\title{
Complications Associated with Bifocal Bone Transport Using Unilateral External Rail Fixator in the Reconstruction of Femur Defects Caused by Infection: Outcome Analysis of 76 Patients
}

\section{Cong Peng}

The First Affiliated Hospital of Xinjiang Medical University

Kai Liu

The First Affiliated Hospital of Xinjiang Medical University

Qi Tian

The First Affiliated Hospital of Xinjiang Medical University

Maimaitiaili Tusunniyazi

The First Affiliated Hospital of Xinjiang Medical University

\section{Weiqi Kong}

The First Affiliated Hospital of Xinjiang Medical University

Haopeng Luan

The First Affiliated Hospital of Xinjiang Medical University

Xiaokang Liu

The First Affiliated Hospital of Xinjiang Medical University

Yan Zhao ( $\nabla$ zhaoyan0325@163.com )

The First Affiliated Hospital of Xinjiang Medical University

\section{Research Article}

Keywords: Bone transport, bone defects, external fixator, infection, complications

Posted Date: July 13th, 2021

DOl: https://doi.org/10.21203/rs.3.rs-670069/v1

License: (c) (i) This work is licensed under a Creative Commons Attribution 4.0 International License. Read Full License 


\section{Abstract}

Background: The purpose of this study is to evaluate the outcomes of single-level bone transport in the treatment of femoral bone defects after radical debridement, which were caused by infections.

Methods: Clinical and radiographic data of patients with infected femoral nonunion treated with a unilateral external fixator (Orthofix limb reconstruction system, LRS) at our hospital from 2010 to 2019 were analyzed retrospectively. The Association for the Study and Application of the Method of llizarov (ASAMI) standard was applied to assess the bone and functional outcomes and postoperative complications evaluated by Paley classification.

Results: Seventy-six cases of infected femoral bone defects (31 proximal, 19 intermediate, and 26 distal) were managed by single-level bone transport using a unilateral external fixator with a mean follow-up time of 30.8 (23 to 41$)$ months. There were 58 men (76.3\%) and 18 women (23.6\%), with a mean age of 38.8 years (range, $23-60$ years). The bone union was achieved in 76 cases with a mean time of 6.9 months (range, 5-8 months). Complications included 29 cases (38.1\%) of pin tract infection, 7 cases $(9.2 \%)$ of muscle contractures, 3 cases $(7.9 \%)$ of joint stiffness, 13 cases $(17.1 \%)$ of axial deviation, 2 cases $(2.6 \%)$ of delayed union, one case $(1.3 \%)$ of nonunion, and none $(0 \%)$ of transport gap re-fracture. One patient (1.3\%) was scheduled for knee arthroplasty when bone transport treatment ended.

Conclusions: Bone transport in the reconstruction of femoral bone defects using unilateral external rail fixator had a satisfactory rate of bone healing and limb function recovery. Complications of distal femoral bone transport were more severe than other sites, but the complication rate was the least of the three. Complications of the proximal were comparable to the intermediate, and soft-tissue-related complications were more likely to occur in the intermediate transport.

\section{Background}

Nonunion of infection has still been a tricky problem for orthopedic surgeons, occurring commonly after open reduction and internal fixation procedures for closed fractures, or external fixation for open fractures [1-9]. Such bone infection is associated with soft tissue loss generally since the long-term course and complex treatment. Inactive infection is described as infection without drainage for at least 3 months [10]. Conversely, nonunion caused by infection is defined as nonunion associated with active drainage, positive deep bacteriological culture or histological proof, osteomyelitis certified by biopsy or clinical, radiographic, and laboratory results [10-12]. The reconstruction of bone defects in the environment of infection remains a great challenge for clinicians, since the complex microbiota and drug resistance, as well as poor vascularity of scar tissue [13-16].

Some reconstruction procedures have been utilized to reconstruct femoral bone defects, including bone grafting, bone transport (distraction osteogenesis) using external fixators, vascularized bone grafting, Masquelet technique $[9,17,18]$. However, there are also limitations and complications in these treatments, such as morbidity at the donor site, stress fractures, and restriction of the size of bone 
defects. Then, how to weigh the efficacy of the above treatments and design individualized treatment plans for patients to reduce postoperative complications, to achieve maximum recovery of function is of great importance for patients' postoperative life.

The technique of bone transport and acute shortening/lengthening based on the principle of distraction osteogenesis, pioneered by llizarov, has become the popular choice gradually for orthopedic surgeons to treat infected femoral nonunion. Among these, all possible pathologies can be handled by bone transport technique simultaneously, such as infection, defects, length, alignment, and union, while allowing functional utilization of the affected limb. Via published articles [6, 19-22], bone transport using external circular fixators or unilateral rail fixators is recommended, especially for bone defects in the lower limb, because of the better stability and maneuverability. The purpose of this study was to evaluate the results of bone transport using an external unilateral rail fixator in the treatment of femoral defects caused by infection, which varied between 4 and $8 \mathrm{~cm}$, and to document the complication rate, external fixation index (EFI), external fixation time (EFT), bone and functional outcomes.

\section{Methods}

\section{Study design}

From January 2008 to December 2019, the medical records and radiographs were evaluated retrospectively of all patients, whose infected bone defects of the femurs were treated by a unilateral external rail fixator (Orthofix limb reconstruction system, Verona,Italy), after written informed consent from participates and approval from the Ethics Committee of our hospital were received. Inclusion criteria as following: femoral bone defect caused by infection; sinus tract or abscess of affected limbs; positive intraoperative culture or histology supporting a deep infection; single-level bone transport was managed for the bone defect. Patients were excluded for those younger than 18 years old, incomplete medical records, poor compliance, other treatments were performed, or follow-up time less than twenty months.

Currently, the pattern of initial injury, previous surgical and medical treatment, associated injury or diseases, the record of antimicrobial utilization, biopsy, or culture results of secretions. In physical examinations, the range of motion (ROM) of hip and knee, and condition of soft tissues and neurovascular were evaluated. The C-reactive protein (CRP) level, white blood cell (WBC) count, procalcitonin, and erythrocyte sedimentation rate (ESR) were examined and documented. The sensitive antibiotics were given to all patients intravenously for 2 weeks depending on the bacteria isolated. When no bacteria were isolated, antibiotic intravenous prophylaxis with a second-generation cephalosporin (cefuroxime $3 \mathrm{~g}$, twice daily) was given for at least 6 weeks before surgery and 1 week after surgery. If bone exposed, the antibiotic compound preparation (sulbactam $2 \mathrm{~g}$ and ampicillin $4 \mathrm{~g}$, three times a day) was applied for at least 1 week.

\section{Surgical technique}


At the first stage, the infected hardware, necrotic bone, and soft tissue of the affected limb were removed totally by at least 9 liters of low-pressure saline irrigation. The infected biopsies and cultures were collected during the surgical procedure. Antibiotic-impregnated cement spacer ( $5 \mathrm{~g}$ vancomycin per $40 \mathrm{~g}$ gentamicin-loaded bone cement, Heraeus, Hanau, Germany) equal in length to the bone defect was then filled into the gap to receive a high level of local antibiotic concentrations. Afterward, the femur was fixed by Orthofix external rail fixator. Depending on the size of the skin loss, the direct suturing under appropriate tension, local propulsive skin flap, or vascularized free flap was utilized to cover it.

The second stage was not initialized until the infection under control, and the skin and soft tissue healed. Patients were positioned supine on the radiolucent table and spinal anesthesia was performed. Sites of bone defects were marked on the skin preoperatively and approached utilizing splitting the major muscle intraoperatively. Following the previous surgical incision, the existing implant was removed and debridement was conducted until the residual bone showed evidence of punctate cortical hemorrhage (paprika sign). External fixators were placed on the proximal and distal femoral fragment in an anteromedial position parallel to the respective joint. Two or three 4.5-mm-diameter Schanz screws were inserted at the level of the lesser trochanter, distal axial line, and the intramedian bone fragment under the guidance of the intraoperative C-arm. Simultaneously, the desired femoral length and alignment were maintained. These screws were angled at right angles to the anatomical axis of the femur. And the level, which approximately $2-3 \mathrm{~cm}$ below the proximal screws of the femur and superior pole of the patella were chosen respectively to be the line of the osteotomy. After the external fixator sliding clamps assembled and the external frame debugged to meet the axis of the femur, the osteotomy was performed with a Gigli saw. The operation time was less than 2 hours in our cohort since the detailed preoperative plans were designed by experienced surgeons, which were based on the films of X-ray, CT scans, and three-dimensional reconstructed images.

\section{Postoperative management}

Distraction osteogenesis was conducted under the introduction of a senior orthopedic surgeon on the sixth day after surgery, commonly at a velocity of $0.25 \mathrm{~mm}$, four times per day. The speed and rhythm of distraction were adjusted to the patient's discomfort and the quality of re-organisms. Full weight-bearing and joint repair were encouraged for patients throughout the stage of distraction. The pin tract care should be instructed to the patients to prevent the reaction of pin tract, such as washed the pin tract daily using an alcohol swab with a mass percentage of $75 \%$. Subsequently, radiography, WBC, ESR, and CRP were examined at $1,3,6,9,12,18$, and 24 months after bone transport. Bone grafting at the docking site was recommended to perform to prevent docking site nonunion when the distraction stage ended.

\section{Data Collection and Outcome Evaluation}

With electronic medical records were authorized, demographic and clinical data, and postoperative complications were recorded. External fixations were removed when regeneration of the bone transport gap was consolidated adequately, as in the presence of three of the four cortices on anteroposterior and lateral radiographs. Artificially, the femur was divided into three parts equally according to the anatomical 
length of the femur, namely, the proximal, intermediate, and distal. Bone union time was defined as the number of months between the end of the distraction stage and the removal of the external frame, which was incorporated with radiography. Also, the external fixation index $(\mathrm{month} / \mathrm{cm})$ was defined as the ratio of the external fixation time to the length of the bone defect.

All patients who met the inclusion criteria were followed up by the special bone transport questionnaires routinely using the smartphone after discharge. These complications were considered respectively as minor resolved without surgically), major (required additional surgery but not affected final treatment), or sequelae (unresolved at the end of treatment) according to Perry. The Association for the Study and Application of the Method of llizarov (ASAMI) standard was applied to assess the bone and functional results.

\section{Statistical analysis}

Data were input in a Microsoft Excel spreadsheet (Redmond, WA, USA) and reported as frequencies and percentages, then analyzed by the SPSS 20.0 software package (Chicago, IL, USA). Of three groups, analysis of variance was used to compare quantitative abnormal variables, and comparisons of categorical variables were performed using the Kruskal-Wallis test. Comparisons between two groups were conducted using the chi-square test or t-test. Statistical significance was $p<0.05$.

\section{Results}

A total of 76 patients who met the criteria were included in this study between January 2008 to December 2019. There were 58 men (76.3\%) and 18 women (23.6\%), with a mean age of 38.8 years (range, $23-60$ years). Seventy-six femoral defects (31 proximal, 19 intermediate, and 26 distal) caused by infection were managed for bifocal bone transport using the unilateral external rail fixator with a mean postoperative follow-up time of 30.8 (23 to 41 ) months. The mechanisms of initial injury of the femur in all patients were summarized in Table 1, included road traffic accidents (46.1\%), falling from height (17.1\%), direct trauma $(22.3 \%)$, or other $(14.4 \%)$. There were 63 cases of open fractures $(82.8 \%)$ and 13 cases of closed $(17.1 \%)$. The average number of surgical operations performed before applying the final frame was 3.2 (range, 1-7). The draining sinus occurred in 43 patients on admission and 17 of them in an inactive sinus scar (quiescent infection). Of the 31 proximal and 26 distal nonunions, one patient, who had previous knee ankyloses, required planned arthrodesis with bone transport. Pain and limitation of limb function were present in two-thirds of patients. With Cierny and Mader's (CM) classification, 51 cases in type $\varangle$, and 25 cases in type $\varangle$. Eleven patients had an oblique plane deformity that required correction, and the bone defects changing from 0 to $7 \mathrm{~cm}$ after radical debridement. Among these patients, positive bacteria isolated was observed in 61 cases $(80.2 \%)$ by the culture test of secretion. Forty-four (57.8\%) were infected with S. aureus, 11 (14.4\%) in P. cuprina, and 6 (7.8\%) with E. coli, none of them in methicillin-resistant $\mathrm{S}$. aureus, MRSA (Table 4). The mean length of all bone defects was $4.6 \mathrm{~cm}$ (range, $3.7-5.6 \mathrm{~cm})$ after the debridement surgery. There was no statistical significance in age $(p=0.105)$, gender $(p=0.647)$, mode of fracture $(p=0.683)$, and duration of osteomyelitis $(p=0.298)$ (Table 2$)$. 
All patients in our cohort were managed by single-level bone transport, and one patient has performed both arthrodesis and bone transport. There were no intraoperative complications such as neurovascular injury or compartment syndrome. The bone transport procedures were performed under the direct operation of an experienced orthopedic surgeon. Antibiotic-impregnated cement was applied in 53 of 76 patients. The infection was eradicated. Total bone union was received in 76 of 76 cases (100\%) with a mean bone union time of 6.9 months (range, 5-8 months). The mean external fixation time was 11.8 months (range, 6-21 months), and the external fixation index was 1.71 months/cm (range, 1.38-2.41 month $/ \mathrm{cm})$. No statistical significance was observed in the length of the bone defect $(p=0.610), E F I$ $(p=0.733)$, and follow-up time $(p=0.382)$, but there were statistically significant in BUT $(p=0.002)$ and EFT $(p<0.001)$ of the three groups (Table 3$)$. Of the bone grade, a statistically significant difference was found (Table 5) between the proximal, intermediate, and distal (excellent/good/fair/poor/failure, 4/21/6/0/0 vs $7 / 8 / 3 / 1 / 0$ vs $5 / 13 / 6 / 2 / 0, p<0.001)$. As to functional grade, there was a statistically significant difference as well between the three locations (excellent/good/fair/poor/failure, 6/23/2/0/0 vs $7 / 10 / 1 / 1 / 0$ vs $7 / 11 / 6 / 2 / 0, p<0.001)$. The whole procedure of single-level bone transport, which was described by this study refers to Figures 1-2.

Of postoperative complications, the most common of them was pin tract infection, and varying degrees of it had been observed in most patients. Twenty-nine patients (38.1\%) developed pin tract infection, 16 cases in grade 1, 9 cases in grade 2, and 4 cases in grade 3 according to Paley's classification. Grade 1 was treated by strengthening dressing change and grade 2 was treated with culture-sensitive oral antibiotics, and strengthen dressing change to keep pin tract clean and dry. Grade 3 was controlled by the replacement of the Schanz screw's location after debridement. There were 7 cases $(9.2 \%)$ of muscle contractures, 3 cases $(7.9 \%)$ of joint stiffness, 13 cases $(17.1 \%)$ of axial deviation, 2 cases $(2.6 \%)$ of delayed union, one case $(1.3 \%)$ of nonunion, and none $(0 \%)$ of transport gap re-fracture (Table 6$)$. Axial deviations were corrected by adjusting the external fixator radiologically under local anesthesia. Muscle contractures were managed by the tension-release surgery to reconstruct function. Delayed unions and nonunion were recovered through surgical treatment of autologous bone graft with internal fixation (Table 7). Finally, the patient associated with knee joint stiffness obtained physical therapy to improve the ROM of the knee under the direction of a senior physiotherapist. And the knee ROM of two was recovered to 10-85 degrees. Unfortunately, the joint stiffness and intractable pain still existed in one patient, and he was scheduled for knee arthroplasty when bone transport treatment ended.

\section{Discussion}

This study aimed to collect and assess the postoperative results and distribution of complications related to bone transport using unilateral external rail fixator in the treatment of femoral bone defects caused by an infection in our hospital, from January 2008 to December 2019. Radical debridement was a prerequisite for bone transport to ensure an infection-free limb. In addition to the skeletal defects produced after debridement, most patients had previous multi-planar deformities, hip and/or knee stiffness, and muscle atrophy caused by the initial injury. The rate of total bone union was $100 \%$, and the per-patient complication was 0.82 . Pin tract infection, muscle contracture, axial deviation, and soft tissue 
incarceration were more likely to occur in the intermediate part of femoral bone transport, and distal bone transport was at high risk of joint stiffness, delayed union, or nonunion. In addition, a higher rate of perpatient complication was observed in the intermediate of femoral bone transport. However, the complications, which caused severe results, were documented in the distal group.

Although there are many types of llizarov technique [1, 3-5, 23], such as acute shortening, segmental bone transport, limb lengthening, etc., the utilization of bone transport for the treatment of infected bone nonunion is recommended by scholars increasingly. In the comparison of other techniques $[17,18]$, such as cancellous bone grafting, free vascularized fibular grafting, or Masquelet technique, bone transport was usually one-stage-surgery, which was able to correct limb length and deformity, allowed utilization of the limb function earlier, and not limited by soft tissue conditions, thus promoted early rehabilitation. However, bone transport is also a great challenge for patients and surgeons since the long and tedious treatment procedure, requiring a supportive collaboration together $[13,15]$. In this study, a new follow-up model was applied, a regular questionnaire and face-to-face video rehabilitation guidance, based on Internet applications. By this platform, satisfactory postoperative management was achieved, and the efficacy of bone transport for infected bony nonunion was certificated. In detail, the mean length of bone defect in the cohort was $4.6 \mathrm{~cm}$ (range, $3.7-5.6 \mathrm{~cm}$ ). The mean time to external fixation was 12.8 months (range, 7-21 months), and the mean external fixation index was 2.46 months/cm (range, 1.75-3.81 months $/ \mathrm{cm}$ ), respectively. The incidence of excellent bone grade and functional grade was $68.4 \%$ and $84.2 \%$ respectively.

Since the potential limitations of the external fixation itself, many surgical methods have been applied to decrease the EFI to avoid complications, then receive better clinical results further [24-30]. Gupta et al. [31] reported a consecutive series of 14 tibial nonunions, which were managed successfully by unilateral external rail fixator combined with locking plates for simultaneous fixation, with a mean defect size of 6.4 $\mathrm{cm}$, mean external fixator index of 21.2 days $/ \mathrm{cm}$, and a per-patient complication incidence of 0.5 . Furthermore, the study published by Gulabi et al.[32] presented a series of five tibial nonunions were treated effectively by bone transport combined with intramedullary nails to avoid wearing external fixators for complications. The treatment technique described above indeed possessed advantages in shortening the EFT and avoiding complications, but not effective enough in accelerating new bone regeneration in the transport gap. Not only, many studies on this field included patients with infected tibial nonunion rather femoral, based on our review of the literature $[1,3,4,23,33-36]$. Hence, the complications of this technique in the treatment of femoral nonunions caused by infection were evaluated by this study, and provide data for the formulation of better clinical strategies.

Over the past decades, the nonunions of the lower extremity with bone loss have been resolved successfully by bone transport techniques using unilateral external rail fixators $[3,13,16,31]$. The bone graft surgery was recommended to perform at the docking site at the end of the distraction stage by many published articles [4, 34, 36-38]. Wan et al.[39] showed a series of 28 femoral bone defects treated by bone transport using a monolateral external fixator, and obtained a good bone healing rate of $92.8 \%$, because of the bone grafting at the docking site. Furthermore, the study published by Yin et al.[22] 
presented a cohort of 110 patients with nonunion of the lower extremity were treated successfully by bone transport, and the bone graft at the docking site was applied as well when the distraction stage ended. In our series, the bone graft was given to the docking site at the end of the distraction stage, and satisfactory bone healing results were obtained.

Although the utilization of bone transport protocols in combination with intramedullary nails is still controversial, the occurrence of complications during bone transport cannot be ignored. The highest incidence of them was still pin tract infection (38.1\%), which occurred in the femur and middle mostly [14, $40,41]$. It may be related to the developed musculature, rich soft tissue, and blood supply of the area. This also explained why the more axial deviations and muscle contractures happen here, in comparison to the distal. Unfortunately, the ability to obtain good bone quality faster was lost by bone transport in the distal as a result. Even the joint stiffness occurred here more and worse knee motion was brought, since the adjacent relationship to knee [15,42-44]. Therefore, the quadriceps plasty methods and their modifications may be a resolution to knee stiffness. Unfortunately, complications were associated with the above methods, including long incisions, scar contracture or skin necrosis, strong skin tension to wound dehiscence, and leg edema, and severe pain in the early postoperative stage. In our cohort, quadriceps plasty was not performed for all patients. In our opinion, quadriceps plasty may be an alternative method to manage knee stiffness, but the value of this procedure is difficult to decide since the complex complications. Conversely, knee arthroplasty was a salvage approach that may be considered for patients with poor knee range of motion and the presence of stubborn pain.

Via published articles $[6,9,20,22,45-48]$, the application of bone transport in the treatment of infected femoral bone defects is more satisfactory than in the tibia, especially in the grade of bone healing. However, the external fixation time, the external fixation index of our cohort was higher than the staged bone transport technique, which combined with internal fixation or acute shortening and re-lengthening [4, 9]. For instance, Sen et al.[9] showed a mean EFI of 31.8days/cm in a cohort of 17 patients treated by the modified technique of acute shortening and re-lengthening. Additionally, Chou et al.[41] presented a mean $\mathrm{EFI}$ of 0.97 months/cm in a series of six infected femoral nonunions managed by the staged protocol of llizarov distraction osteogenesis and intramedullary nailing. In this study, good bone healing outcomes were also obtained despite the absence of applying a combined intramedullary nail treatment modality as studied above. That may be the result of detailed preoperative planning, skillful mastery of the external fixation framework, and rehabilitation training started on the second postoperative day. The novel combined technique described above can be a good choice to improve the stability of the distal femoral docking site, thereby preventing delayed union and nonunion. But the risk of multiple operations' complications cannot be underestimated, such as long incisions and extensive scars.

Last, several limitations were in this study, which was associated with its retrospective and single-center design nature. First of all, there is no mature algorithm for the resolution of complications in different bone transport locations of the femur. In addition, there is a lack of case series with the postoperative complications of infected femoral nonunions treated with bone transport. Therefore, a prospective study of more samples and multi-center is of more clinical significance. 


\section{Conclusion}

By comparing the postoperative results and complications of bone transport in different locations of the femur, the following conclusions were summarized. Complications of distal femoral bone transport were more severe than other sites, but the complication rate was the least of the three. Complications of the proximal were comparable to the intermediate, and soft-tissue-related complications were more likely to occur in the intermediate transport. The bone healing and functional outcomes were affected directly by these complications in the period of bone transport. Bone grafting surgery to the docking site can prevent delayed union or nonunion effectively. It's worthy to understand the incidence and distribution of complications at three different locations was helpful to formulate targeted measures to deal with postoperative complications, and can promote bone healing effectively.

\section{Declarations}

\section{Acknowledgments}

Not applicable.

\section{Author Contributions}

Cong Peng: Conducted the study. Collected, analyzed, and interpreted the data. Wrote the manuscript.

Kai Liu: Designed the study, and interpreted the data, and edited the manuscript.

Qi Tian: Planned the project. Interpreted the data.

Maimaitiaili Tusunniyazi: Edited the manuscript.

Weiqi Kong: Interpreted the data.

Haopeng Luan: Edited the manuscript.

Xiaokang Liu: Reviewed the manuscript.

Yan Zhao: Planned the project. Reviewed the manuscript.

\section{Funding information}

This study was not funded by any foundation.

\section{Availability of data and materials}

The data sets generated and analyzed during the current study are not publicly available due to restrictions on ethical approvals involving patient data and anonymity but can be obtained from the appropriate authors as reasonably required. 
Ethics approval and consent to participate

This retrospective study was approved by the Ethics Committee of The First Affiliated Hospital of Xinjiang Medical University and carried out in accordance with the ethical standards set out in the Helsinki Declaration. Informed consent was received from all participating.

Competing interests

The authors declare that they have no conflict of interest.

Consent for publication

Not applicable.

\section{Disclosure}

The authors report no proprietary or commercial interest in any product mentioned or concept discussed in this article.

\section{References}

1. Van Hentenryck M: Growing Pains. JAMA 2020:324, 8.

2. Matsuhashi M, Saito T, Noda T, Uehara T, Shimamura Y, Ozaki T: Treatment for postoperative infection of pathological femoral fracture after radiotherapy: two case reports and review of the literature. ARCH ORTHOP TRAUM SU 2020.

3. Bezstarosti H, Metsemakers WJ, van Lieshout EMM, Voskamp LW, Kortram K, McNally MA, Marais LC, Verhofstad MHJ: Management of critical-sized bone defects in the treatment of fracture-related infection: a systematic review and pooled analysis. ARCH ORTHOP TRAUM SU 2020.

4. Blázquez-Carmona P, Mora-Macías J, Morgaz J, Fernández-Sarmiento JA, Domínguez J, Reina-Romo $\mathrm{E}$ : Mechanobiology of Bone Consolidation During Distraction Osteogenesis: Bone Lengthening Vs. Bone Transport. ANN BIOMED ENG 2020.

5. El-Rosasy MA, Ayoub MA: Traumatic Composite Bone and Soft Tissue Loss of the Leg: RegionSpecific Classification and Treatment Algorithm. INJURY 2020:S20-S1383.

6. Corona PS, Ramirez-Nuñez LJ, Amat C, Carrera L: Outcome of oscillating saw open osteotomy in two-stage lower extremity bone transport with monolateral frame. INJURY 2017, 48(10):2285-2291.

7. Peschiera V, Staletti L, Cavanna M, Saporito M, Berlusconi M: Predicting the failure in distal femur fractures. INJURY 2018, 49:S2-S7.

8. Raven TF, Moghaddam A, Ermisch C, Westhauser F, Heller R, Bruckner T, Schmidmaier G: Use of Masquelet technique in treatment of septic and atrophic fracture nonunion. INJURY 2019, 50:40-54. 
9. Sen C, Demirel M, Sağlam Y, Balcı HI, Eralp L, Kocaoğlu M: Acute shortening versus bone transport for the treatment of infected femur non-unions with bone defects. INJURY 2019, 50(11):2075-2083.

10. Cierny GR, Mader JT, Penninck JJ: A clinical staging system for adult osteomyelitis. Clin Orthop Relat Res 2003(414):7-24.

11. Bell A, Templeman D, Weinlein JC: Nonunion of the Femur and Tibia. ORTHOP CLIN N AM 2016, 47(2):365-375.

12. Nicholson JA, Makaram N, Simpson A, Keating JF: Fracture nonunion in long bones: A literature review of risk factors and surgical management. INJURY 2020.

13. Borzunov DY, Shastov AL: Mechanical solutions to salvage failed distraction osteogenesis in large bone defect management. INT ORTHOP 2019, 43(5):1051-1059.

14. Obremskey WT, Metsemakers W, Schlatterer DR, Tetsworth K, Egol K, Kates S, McNally M: Musculoskeletal Infection in Orthopaedic Trauma. Journal of Bone and Joint Surgery 2020, 102(10):e41-e44.

15. Aktuglu K, Erol K, Vahabi A: Ilizarov bone transport and treatment of critical-sized tibial bone defects: a narrative review. Journal of Orthopaedics and Traumatology 2019, 20(1).

16. Papakostidis C, Bhandari M, Giannoudis PV: Distraction osteogenesis in the treatment of long bone defects of the lower limbs: effectiveness, complications and clinical results, a systematic review and meta-analysis. BONE JOINT J 2013, 95-B(12):1673-1680.

17. Klein C, Monet M, Barbier V, Vanlaeys A, Masquelet AC, Gouron R, Mentaverri R: The Masquelet technique: Current concepts, animal models, and perspectives. J TISSUE ENG REGEN M 2020.

18. Deng AD, Innocenti M, Arora R, Gabl M, Tang JB: Vascularized Small-Bone Transfers for Fracture Nonunion and Bony Defects. CLIN PLAST SURG 2020, 47(4):501-520.

19. Sangkaew C: Distraction osteogenesis of the femur using conventional monolateral external fixator. ARCH ORTHOP TRAUM SU 2008, 128(9):889-899.

20. Ma C, Chiu Y, Tu Y, Yen C, Wu C: Three-stage treatment protocol for recalcitrant distal femoral nonunion. ARCH ORTHOP TRAUM SU 2017, 137(4):489-498.

21. Xu Y, Fan X, He X, Wen H: Correction to: Reconstruction of massive tibial bone and soft tissue defects by trifocal bone transport combined with soft tissue distraction: experience from 31 cases. $B M C$ MUSCULOSKEL DIS 2021, 22(1).

22. Yin P, Zhang L, Li T, Zhang L, Wang G, Li J, Liu J, Zhou J, Zhang Q, Tang P: Infected nonunion of tibia and femur treated by bone transport. J ORTHOP SURG RES 2015, 10(1).

23. Wen $\mathrm{H}$, Zhu S, Li C, Xu Y: Bone transport versus acute shortening for the management of infected tibial bone defects: a meta-analysis. BMC MUSCULOSKEL DIS 2020, 21(1).

24. Gulabi D, Erdem M, Cecen GS, Avci CC, Saglam N, Saglam F: llizarov fixator combined with an intramedullary nail for tibial nonunions with bone loss: is it effective? Clin Orthop Relat Res 2014, 472(12):3892-3901. 
25. Deng AD, Innocenti M, Arora R, Gabl M, Tang JB: Vascularized Small-Bone Transfers for Fracture Nonunion and Bony Defects. CLIN PLAST SURG 2020, 47(4):501-520.

26. Nicholson JA, Makaram N, Simpson A, Keating JF: Fracture nonunion in long bones: A literature review of risk factors and surgical management. INJURY 2020.

27. Sen C, Demirel M, Sağlam Y, Balcı HI, Eralp L, Kocaoğlu M: Acute shortening versus bone transport for the treatment of infected femur non-unions with bone defects. INJURY 2019, 50(11):2075-2083.

28. Chou P, Lin H, Su Y, Chiang C, Chang M, Chen C: Staged protocol for the treatment of chronic femoral shaft osteomyelitis with llizarov's technique followed by the use of intramedullary locked nail. $J \mathrm{CHIN}$ MED ASSOC 2017, 80(6):376-382.

29. Ma C, Chiu Y, Tu Y, Yen C, Wu C: Three-stage treatment protocol for recalcitrant distal femoral nonunion. ARCH ORTHOP TRAUM SU 2017, 137(4):489-498.

30. Davda K, Heidari N, Calder P, Goodier D: 'Rail and Nail' bifocal management of atrophic femoral nonunion. BONE JOINT J 2018, 100-B(5):634-639.

31. Gupta S, Malhotra A, Mittal N, Garg SK, Jindal R, Kansay R: The management of infected nonunion of tibia with a segmental defect using simultaneous fixation with a monorail fixator and a locked plate. BONE JOINT J 2018, 100-B(8):1094-1099.

32. Gulabi D, Erdem M, Cecen GS, Avci CC, Saglam N, Saglam F: Ilizarov fixator combined with an intramedullary nail for tibial nonunions with bone loss: is it effective? Clin Orthop Relat Res 2014, 472(12):3892-3901.

33. Li Y, Shen S, Xiao Q, Wang G, Yang H, Zhao H, Shu B, Zhuo N: Efficacy comparison of double-level and single-level bone transport with Orthofix fixator for treatment of tibia fracture with massive bone defects. INT ORTHOP 2020, 44(5):957-963.

34. Biz C, Crimì A, Fantoni I, Vigo M, lacobellis C, Ruggieri P: Functional outcome and complications after treatment of comminuted tibial fractures or deformities using llizarov bone transport: a single-center study at 15- to 30-year follow-up. ARCH ORTHOP TRAUM SU 2020.

35. Yushan M, Ren P, Abula A, Alike Y, Abulaiti A, Ma C, Yusufu A: Bifocal or Trifocal (Double-Level) Bone Transport Using Unilateral Rail System in the Treatment of Large Tibial Defects Caused by Infection: A Retrospective Study. ORTHOP SURG 2020, 12(1):184-193.

36. Testa G, Vescio A, Aloj DC, Costa D, Papotto G, Gurrieri L, Sessa G, Pavone V: Treatment of Infected Tibial Non-Unions with Ilizarov Technique: A Case Series. J CLIN MED 2020, 9(5):1352.

37. Szelerski A, Żarek S, Górski R, Mochocki K, Górski R, Morasiewicz P, Małdyk P: Surgical treatment outcomes of the llizarov and internal osteosynthesis methods in posttraumatic pseudarthrosis of the tibia-a retrospective comparative analysis. J ORTHOP SURG RES 2020, 15(1).

38. Thakeb MF, Fayyad TA, ElGebeily MA, Diab RA, El Zahlawy H, Sharafeldin MS, Al Kersh MA: Bifocal Compression-Distraction for Combined Bone and Soft-Tissue Defects in Post-traumatic Tibial Nonunion. J ORTHOP TRAUMA 2019, 33(10):e372-e377.

39. Wan J, Ling L, Zhang X, Li Z: Femoral bone transport by a monolateral external fixator with or without the use of intramedullary nail: a single-department retrospective study. European journal of 
orthopaedic surgery \& traumatology 2013, 23(4):457-464.

40. Papakostidis C, Bhandari M, Giannoudis PV: Distraction osteogenesis in the treatment of long bone defects of the lower limbs: effectiveness, complications and clinical results, a systematic review and meta-analysis. BONE JOINT J 2013, 95-B(12):1673-1680.

41. Chou P, Lin H, Su Y, Chiang C, Chang M, Chen C: Staged protocol for the treatment of chronic femoral shaft osteomyelitis with llizarov's technique followed by the use of intramedullary locked nail. $J$ CHIN MED ASSOC 2017, 80(6):376-382.

42. Liu K, Cai F, Liu Y, Abulaiti A, Ren P, Yusufu A: Risk factors of ankle osteoarthritis in the treatment of critical bone defects using ilizarov technique. BMC Musculoskelet Disord 2021, 22(1):339.

43. Borzunov DY, Kolchin SN, Malkova TA: Role of the llizarov non-free bone plasty in the management of long bone defects and nonunion: Problems solved and unsolved. World J Orthop 2020, 11(6):304318.

44. Liu Y, Yushan M, Liu Z, Liu J, Ma C, Yusufu A: Complications of bone transport technique using the llizarov method in the lower extremity: a retrospective analysis of $\mathbf{2 8 2}$ consecutive cases over $\mathbf{1 0}$ years. BMC Musculoskelet Disord 2020, 21(1):354.

45. Large TM, Adams MR, Loeffler BJ, Gardner MJ: Posttraumatic Avascular Necrosis After Proximal Femur, Proximal Humerus, Talar Neck, and Scaphoid Fractures. J AM ACAD ORTHOP SUR 2019, 27(21):794-805.

46. Liska F, Voss A, Imhoff FB, Willinger L, Imhoff AB: Nonunion and delayed union in lateral open wedge distal femoral osteotomies-a legitimate concern? INT ORTHOP 2018, 42(1):9-15.

47. Koso RE, Terhoeve C, Steen RG, Zura R: Healing, nonunion, and re-operation after internal fixation of diaphyseal and distal femoral fractures: a systematic review and meta-analysis. INT ORTHOP 2018, 42(11):2675-2683.

48. Borzunov DY, Shastov AL: Mechanical solutions to salvage failed distraction osteogenesis in large bone defect management. INT ORTHOP 2019, 43(5):1051-1059.

\section{Tables}

Table 1 Summary of the mechanisms of initial injury.

\begin{tabular}{lll} 
Mechanism & Number $(\mathrm{n}=62)$ & Percentage (\%) \\
\hline Road traffic accident & 35 & 46.1 \\
\hline Falling from height & 13 & 17.1 \\
\hline Direct trauma & 17 & 22.3 \\
\hline Others & 11 & 14.4
\end{tabular}


Table 2 Demographic of patients.

\begin{tabular}{lllll} 
Variables & $\begin{array}{l}\text { Proximal } \\
(\mathrm{n}=31)\end{array}$ & $\begin{array}{l}\text { Intermediate } \\
(\mathrm{n}=19)\end{array}$ & $\begin{array}{l}\text { Distal } \\
(\mathrm{n}=26)\end{array}$ & $\begin{array}{l}\mathrm{P} \\
\text { value }\end{array}$ \\
\hline Age (years) & $\begin{array}{l}41(23- \\
55)\end{array}$ & $38(22-51)$ & $\begin{array}{l}39(20- \\
57)\end{array}$ & 0.105 \\
\hline Gender (male, female) & $23 \mathrm{M} 8 \mathrm{~F}$ & $16 \mathrm{M} 3 \mathrm{~F}$ & $19 \mathrm{M} 7 \mathrm{~F}$ & 0.647 \\
\hline Mode of fracture (open, closed) & $2506 \mathrm{C}$ & $1702 \mathrm{C}$ & $2105 \mathrm{C}$ & 0.683 \\
\hline $\begin{array}{l}\text { Duration of post-traumatic osteomyelitis } \\
\text { (months) }\end{array}$ & $\begin{array}{l}30(22- \\
32)\end{array}$ & $29(22-35)$ & $\begin{array}{l}31(16- \\
41)\end{array}$ & 0.298 \\
\hline
\end{tabular}

Table 3 Postoperative data of patients.

\begin{tabular}{lllll} 
Variables & $\begin{array}{l}\text { Proximal } \\
(\mathrm{n}=31)\end{array}$ & Intermediate $(\mathrm{n}=19)$ & $\begin{array}{l}\text { Distal } \\
(\mathrm{n}=26)\end{array}$ & P value \\
\hline Length of bone defect $(\mathrm{cm})$ & $4.5(4.0-5.4)$ & $4.8(4.1-5.5)$ & $4.7(3.6-5.7)$ & 0.610 \\
\hline BUT(month) & $9.5(6-13)$ & $13.6(7-19)$ & $11.4(8-15)$ & 0.002 \\
\hline EFT (month) & $10.8(7-15)$ & $14.1(8-21)$ & $13.6(9-19)$ & $<0.001$ \\
\hline EFI (month/cm) & $2.36(1.75-2.78)$ & $2.31(1.95-3.81)$ & $2.73(2.5-3.33)$ & 0.733 \\
\hline Follow-up time(month) & $31(24-41)$ & $30(23-39)$ & $29(24-33)$ & 0.382
\end{tabular}

BUT: bone union time, EFT: external fixation time, EFI: external fixation index.

Table 4 Summary of patients with isolated organism and final outcome 


\begin{tabular}{lll} 
Organism & $\begin{array}{l}\text { Number of } \\
\text { cases }\end{array}$ & Outcome \\
\hline S. aureus & $44(57.8 \%)$ & Resolved by intravenous antibiotic therapy. \\
\hline $\begin{array}{l}\text { S. } \\
\text { epidermidis }\end{array}$ & $11(14.4 \%)$ & Resolved by intravenous antibiotic therapy. \\
\hline E. coli & $6(7.8 \%)$ & Resolved by oral antibiotics. \\
\hline MRSA & $0(0 \%)$ & - \\
\hline $\begin{array}{l}\text { None } \\
\text { isolated }\end{array}$ & $15(19.2 \%)$ & $\begin{array}{l}\text { Resolved by oral antibiotics combined with intravenous } \\
\text { antibiotics. }\end{array}$ \\
\hline
\end{tabular}

MRSA, methicillin-resistant Staphylococcus aureus.

Table 5 Outcomes of ASAMI scores in different locations

\begin{tabular}{lllllll} 
ASAMI & Location & Excellent & Good & Fair & Poor & Failure \\
\hline Bone grade $^{\#}$ & Proximal & 4 & 21 & 6 & 0 & - \\
\hline & Intermediate & 7 & 8 & 3 & 1 & - \\
\hline & Distal & 5 & 13 & 6 & 2 & - \\
\hline Function grade $^{*}$ & Proximal & 6 & 23 & 2 & 0 & - \\
\hline & Intermediate & 7 & 10 & 1 & 1 & - \\
\hline & Distal & 7 & 11 & 6 & 2 & -
\end{tabular}

P, proximal. I, intermediate. Distal, D.

\# P vs $D p=0.571$, I vs $D p<0.001$, I vs $D p<0.001$

* $P$ vs I $p<0.001$, I vs $D$ p $=0.643$, P vs $D p<0.001$

Table 6 Complications in the period of bone transport 
Complication Proximal Intermediate $(n=19)$ Distal

\begin{tabular}{llll} 
& $(\mathrm{n}=31)$ & $(\mathrm{n}=26)$ \\
\hline Pin tract infection & 11 & 13 & 5 \\
\hline Muscle contractures & 3 & 4 & 0 \\
\hline Joint stiffness & 1 & 0 & 2 \\
\hline Axial deviation & 4 & 7 & 2 \\
\hline Soft tissue incarceration & 1 & 3 & 2 \\
\hline Neurological injury & 0 & 0 & 0 \\
\hline Delayed union & 0 & 1 & 2 \\
\hline Nonunion & 0 & 0 & 1 \\
\hline Transport gap re-fracture & 0 & 0 & 0
\end{tabular}

Table 7 The treatment outcomes of complications (all patients)

\begin{tabular}{llll} 
Complication & $\begin{array}{l}\text { Number of } \\
\text { patients }\end{array}$ & Treatment & Outcome \\
\hline $\begin{array}{l}\text { Pin tract } \\
\text { infection }\end{array}$ & $29(38.1 \%)$ & $\begin{array}{l}\text { strengthen dressing change, culture-sensitive oral } \\
\text { antibiotics or surgical intervention }\end{array}$ & Union \\
\hline $\begin{array}{l}\text { Muscle } \\
\text { contracture }\end{array}$ & $7(9.2 \%)$ & surgical intervention under spinal anesthesia & Union \\
\hline $\begin{array}{l}\text { Knee joint } \\
\text { stiffness }\end{array}$ & $3(7.9 \%)$ & Physical therapy & Union \\
\hline $\begin{array}{l}\text { Axial } \\
\text { deviation }\end{array}$ & $13(17.1 \%)$ & surgical intervention under local anesthesia & Union \\
\hline $\begin{array}{l}\text { Delayed } \\
\text { union }\end{array}$ & $2(2.6 \%)$ & surgical intervention under general anesthesia & Union \\
\hline Nonunion & $1(1.3 \%)$ & surgical intervention under general anesthesia & Union
\end{tabular}

\section{Figures}




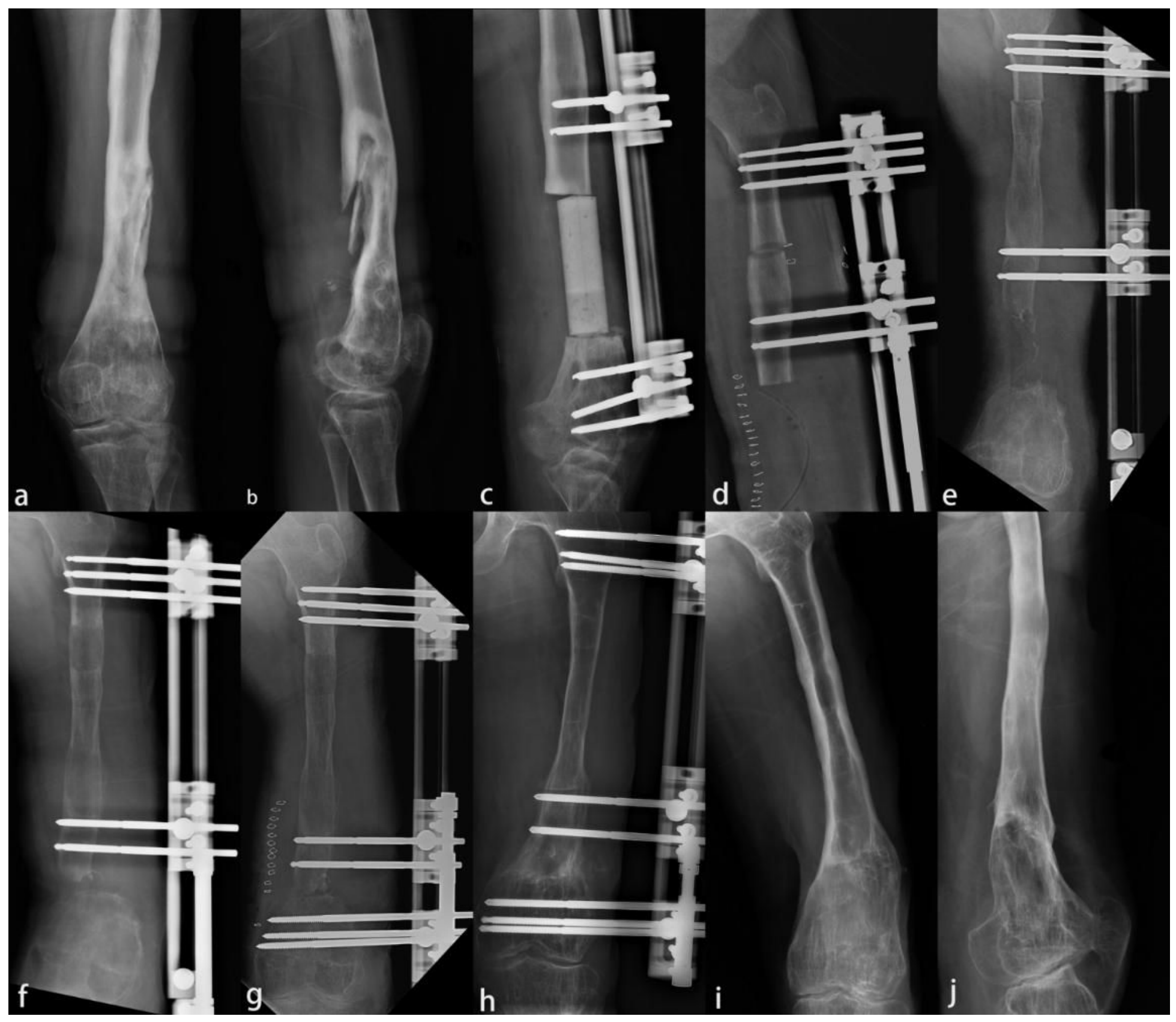

Figure 1

A 20-year-old male with post-traumatic osteomyelitis of the left femur was treated with single-level bone transport using external rail fixator. $(a, b)$ Osteomyelitis of the left femur caused by post-traumatic infection presented by X-ray films. (c, d, e) Resection of infected bone with $7.8 \mathrm{~cm}$ defect, the antibioticimpregnated cement spacer was filled and removed until infection was controlled, then treated by singlelevel bone transport. (f) Radiological films of 6 weeks in the distraction stage. $(\mathrm{g}, \mathrm{h})$ Bone transport completed with good regenerate consolidation, and docking union was achieved at four postoperative months. (i, j) External frame was removed with excellent bone result represented by view of X-ray at six postoperative months. 